\title{
ON THE STRUCTURE OF OPTIMAL ORDERING POLICIES FOR STOCHASTIC INVENTORY SYSTEMS WITH MINIMUM ORDER QUANTITY
}

\author{
Yao Zhao and Michael N. Katehakis \\ Department of Management Science and Information Systems \\ Rutgers University, The State University of New Jersey \\ Newark, NJ 07102-1895 \\ E-mail:yaozhao@andromeda.rutgers.edu; mnk@andromeda.rutgers.edu
}

\begin{abstract}
We study a single-product periodic-review inventory model in which the ordering quantity is either zero or at least a minimum order size. The ordering cost is a linear function of the ordering quantity, and the demand in different time periods are independent random variables. The objective is to characterize the inventory policies that minimize the total discounted ordering, holding, and backorder penalty costs over a finite time horizon. We introduce the concept of an $M$-increasing function. These functions allow us to characterize the optimal inventory policies everywhere in the state space outside of an interval for each time period. Furthermore, we identify easily computable upper bounds and asymptotic lower bounds for these intervals. Finally, examples are given to demonstrate the complex structure of the optimal inventory policies.
\end{abstract}

\section{INTRODUCTION}

Minimum Order Quantity (MOQ) is widely used in many industries (e.g., apparel, pharmaceutical, and consumer packaged products). In these industries, customers (e.g., distributors or retailers) typically have two choices, either not to order from their suppliers or to order at least a minimum quantity, namely the MOQ. This MOQ provides a way for the suppliers to achieve economies of scale in production and transportation.

A well-known example of MOQ is the fashion sport ski-wear distributor Sport Obermeyer [7]. Sport Obermeyer outsources almost all of its production to Hong 
Kong and China to reduce production variable cost. Due to the economy of scale in mass production, the manufacturing facilities require a minimum ordering quantity of 600 garments in Hong Kong and 1200 garments in China per order. The MOQ constraints apply to a particular item or a group of items, such as all colors of a particular style. MOQ is not only required in fashion industries where products are subject to obsolescence (thus distributors and retailers only place orders for a few items), it also applies to many repeatedly ordered items. For instance, Home Depot and Wal-Mart have to honor the MOQs specified by the suppliers for many nonseasonal items they carry. When the suppliers require MOQ, they typically do not charge a fixed ordering cost (which is accounted by the MOQ), but charge the same unit price per item. Therefore, the customers' ordering cost is a linear function of the ordering quantity.

Minimum order quantity stands as a substantial challenge to managing supply chains efficiently. As illustrated by the numerical examples of Fisher and Raman [7], MOQ requires the distributors or retailers (hereafter we use retailer and distributor interchangeably) to order either none or many. Thus, it reduces the retailers' flexibility of responding to demand and eventually increases the retailers' inventory costs or reduces their service levels. To provide tools and guidance for companies to effectively manage inventory when they face MOQs from their suppliers, we model, in this article, this type of problem as stochastic dynamic programs and investigate the structure of the optimal policy for the single-product, periodic-review inventory system with MOQ in a finite time horizon.

There is a large body of literature in the general areas of production and inventory management incorporating the impact of the economy of scale. However, most efforts have been devoted to models with fixed ordering costs and fixed batch sizes; little attention has been paid to MOQs. Scarf [16] proved that an $(s, S)$ policy is optimal for periodic-review, single-product inventory systems with fixed ordering cost and general demand distributions. An alternative proof is provided by Veinott [20] under a different set of assumptions. An excellent review of stochastic inventory systems with fixed ordering cost can be found in Heyman and Sobel [9]. For systems with fixed batch sizes, Veinott [19] first proved that a reorder point/batchtransfer policy is optimal. In this policy, if the inventory level drops below the reorder point, orders are placed in batches to raise the inventory level to the smallest value above the reorder point; if the inventory level is above the reorder point, no order is placed. Chen [4] proved that the policy is optimal for multiechelon inventory systems under some regularity conditions. Karlin [10] analyzed a single-period stochastic inventory system in which a fixed amount is added to stock whenever a decision to increase stock is made. This model has applications in production systems in which the difference between the cost of producing up to the capacity and the cost of producing any number of units less than the capacity is negligible. Karlin [10] specified the conditions under which the optimal inventory policy can be characterized by a single critical number.

Chan and Muckstadt [3] studied a stochastic production-inventory system in which the production quantity is constrained by a minimum and a maximum level 
in each time period, namely, the production smoothing problem. They characterized the optimal production policy in finite and infinite time horizons under the discounted cost criterion. This production smoothing problem is different from the model studied here because in the former, the action of zero production is absent and, hence, the action sets are connected and compact. In the model studied here, the order quantity is either zero or at least the MOQ; therefore, the action sets are disjoint and unbounded. As we will see later, these disjoint action sets significantly complicate the structure of the optimal policies. Other related work in this general area includes that of Axsater [1], De Bodt and Graves [5], Federgruen and Katalan [6], Gallego and Wolf [8], Karmarkar [11], Lee and Moinzadeh [13], Porteus [14], Sethi and Cheng [17], Zheng and Federgruen [22], Zipkin [23], and Song and Yao [18].

The work of Fisher and Raman [7] is perhaps the first article explicitly considering MOQ in stochastic inventory systems. They considered a distributor managing multiple products in two time periods. If the distributor decides to order, the order sizes must be larger than or equal to the MOQs for each time period and less than the supplier's production capacity. They solved the optimal order quantity problem using stochastic programming, and they quantified the impact of MOQ on the distributor's inventory costs for the case of two time periods. Katehakis and Bradford [12] considered a related single-period, multilocation distribution system with both minimum and maximum order quantity constraints. To the best of our knowledge, there are almost no studies on the multiperiod inventory systems with MOQs. Indeed, the structure of the optimal inventory policy is not known even for the simplest type of such systems with a single product and unlimited production capacity.

This article is organized as follows: In Section 2 the model and notation are presented. In Section 3 we establish, in Theorem 3.10, the form of the optimal policy outside of an interval for each time period. In Lemma 3.9, Lemma 3.12, and Theorem 3.13 we provide the upper bound and asymptotic lower bound for this interval in each time period. Examples are given to demonstrate the complex structure of the optimal policy. Section 4 concludes the article by pointing out future research directions.

\section{THE MODEL AND FORMULATION}

We consider a single retailer managing a single product and reviewing its inventory periodically. External demand is stochastic and a minimum order quantity, $M$, is required from the supplier.

The sequence of events is as follows. At the beginning of a time period, the retailer reviews the inventory and makes order decisions. If he orders, the order size must be at least $M$ units and it is immediately filled. At the end of the time period, demand is realized and the retailer fills the demand from on-hand stock. Any excessive demand is backlogged. Our analysis can be easily extended to systems with constant replenishment lead times following the standard argument in Heyman and Sobel [9]. 
Let $N$ be an integer that represents the number of time periods in the planning horizon. We index time periods $n=1,2, \ldots, N$, where $N$ is the first time period in the planning horizon and 1 is the last. Let $c, \beta(0<\beta<1), h$, and $\pi$ be the unit ordering cost, the time discounted factor for each time period, the unit inventory holding cost per time period, and the unit backorder penalty per time period, respectively. We use $D_{n}$ to denote the external demand in time period $n$, and it is assumed that the $D_{n}$ 's are independent with finite means $E\left(D_{n}\right)$. Throughout this article, we assume $\pi>c$.

Following Markov Decision Process (MDP) notations, let $S$ be the continuous state space for inventory position $x$, at the beginning of a time period, and $y-x$ be the amount ordered in that time period. $y \in A_{x}$, where $A_{x}=\{x\} \cup[x+M,+\infty)$ is the set of feasible actions. Let $\xi(x, y, D)=y-D$ be the transition function, where $D$ is the demand.

It is easy to verify that the expected inventory and ordering cost in one time period given that the period starts with an inventory position $x$ and orders in that period $y-x$ items can be written as follows:

$$
c(y-x)+E(L(y, D)),
$$

where $E(\cdot)$ is the expectation with respect to $D$ and

$$
L(y, D)=h(y-D)^{+}+\pi(D-y)^{+} .
$$

Let $U_{n}(x)$ be the minimum total inventory and ordering costs from the beginning of $n$th time period until the end of the planning horizon, given an $n$th period initial inventory position $x$. Clearly,

$$
\begin{aligned}
& U_{1}(x)=\inf _{y \in A_{x}}\left\{c(y-x)+E\left(L\left(y, D_{1}\right)\right)\right\}, \\
& U_{n}(x)=\inf _{y \in A_{x}}\left\{c(y-x)+E\left(L\left(y, D_{n}\right)\right)+\beta E\left(U_{n-1}\left(y-D_{n}\right)\right)\right\}, \quad n=2, \ldots, N .
\end{aligned}
$$

The salvage value of the unsold products at the end of the planning horizon is zero (i.e., $\left.U_{0}(x) \equiv 0\right)$. Observe that if $M=0$, Eq. (1) reduces to the well-known inventory model of zero fixed ordering cost [15].

Define $V_{n}(x)=U_{n}(x)+c x, \forall n$; we can simplify Eq. (1) as follows:

$$
\begin{aligned}
& V_{1}(x)=\inf _{y \in A_{x}}\left\{c y+E\left(L\left(y, D_{1}\right)\right)\right\}, \\
& V_{n}(x)=\inf _{y \in A_{x}}\left\{c(1-\beta) y+E\left(L\left(y, D_{n}\right)\right)+\beta E\left(V_{n-1}\left(y-D_{n}\right)\right)\right\}+\beta c E\left(D_{n}\right), \\
& n=2, \ldots, N .
\end{aligned}
$$


For simplicity, we define $g_{n}(y)=c(1-\beta) y+E\left(L\left(y, D_{n}\right)\right)$ for $n \geq 2$ and $g_{1}(y)=$ $c y+E\left(L\left(y, D_{1}\right)\right)$; we let $H_{n}(y)=g_{n}(y)+\beta E\left(V_{n-1}\left(y-D_{n}\right)\right), \forall n \geq 2$, and $H_{1}(y)=$ $g_{1}(y)$. We can rewrite Eq. (2) as

$$
\begin{aligned}
& V_{1}(x)=\inf \left\{H_{1}(x) ; H_{1}(y): y \geq x+M\right\}, \\
& V_{n}(x)=\inf \left\{H_{n}(x) ; H_{n}(y): y \geq x+M\right\}+\beta c E\left(D_{n}\right), \quad n=2, \ldots, N .
\end{aligned}
$$

We can prove the following proposition by induction.

Proposition 2.1: If $M \leq M^{\prime}$ while everything else is identical, then $U_{n}(x) \leq U_{n}^{\prime}(x)$, $\forall x$, where $U\left(U^{\prime}\right)$ corresponds to $M\left(M^{\prime}\right.$, respectively).

The proof is based on the observation that $A_{x} \supseteq A_{x}^{\prime}$, where $A\left(A^{\prime}\right)$ corresponds to $M$ ( $M^{\prime}$, respectively). We omit the details of the proof.

\section{STRUCTURE OF THE OPTIMAL POLICY}

We first focus on the last time period in the planning horizon.

Definition 3.1: Consider two continuous functions, $\phi(y)$ and $\psi(y)$, where $y \in$ $(-\infty,+\infty)$. If there exists a positive $\bar{\epsilon}, \bar{\delta}$, and $a \omega=[a, b]$ where $a$ and $b$ are finite constants, so that $\phi(y)=\psi(y), \forall y \in[a, b]$, and $(\phi(a-\epsilon)-\psi(a-\epsilon))$ $(\phi(b+\delta)-\psi(b+\delta))<0$ for any $0<\epsilon \leq \bar{\epsilon}$ and $0<\delta \leq \bar{\delta}$, then we define a to be a junction.

Intuitively, a junction is the minimum point of an interval in which $\phi(y)-$ $\psi(y)=0$ and beyond which, $\phi(y)-\psi(y)$ changes sign.

LEMMA 3.2: Given any convex function $\phi(y), y \in(-\infty,+\infty)$, that satisfies $\phi(y) \rightarrow$ $+\infty$ as $|y| \rightarrow+\infty$, define $y^{*}$ to be the smallest value at which $\phi(y)$ reaches the global minimum.

1. There exists a unique and finite junction $y_{0}$ between $\phi(y)$ and $\phi(y+M)$.

2.

$$
\begin{aligned}
& \phi(x) \leq \phi\left(x+M^{\prime}\right), \quad \forall x>y_{0}, \forall M^{\prime} \geq M, \\
& \phi(x) \geq \phi(x+M), \quad \forall x<y_{0} .
\end{aligned}
$$

3. $y^{*}-M<y_{0} \leq y^{*}$.

PROOF:

1. Observe that $\phi(x)$ is continuous (due to convexity), that there exists a $y_{1}>-\infty$ so that $\phi\left(y_{1}\right)>\phi\left(y_{1}+M\right)$, and that there exists a $y_{2}<+\infty$ so that $\phi\left(y_{2}\right)<\phi\left(y_{2}+M\right)$. It follows from the Intermediate Value Theorem of continuous functions that there must exist a junction between $\phi(y)$ and $\phi(y+M)$, and all junctions between $\phi(y)$ and $\phi(y+M)$ must be finite. 
To show the uniqueness of the junction, we prove that if $\phi\left(y_{1}\right)=$ $\phi\left(y_{1}+M\right)$ and $\phi\left(y_{2}\right)=\phi\left(y_{2}+M\right)$ for two points $y_{1}<y_{2}$, then $\phi(y)=$ $\phi(y+M), \forall y \in\left(y_{1}, y_{2}\right)$.

For any $y \in\left(y_{1}, y_{2}\right)$, the convexity of $\phi(y)$ implies that

$$
\frac{\phi(y)-\phi\left(y_{1}\right)}{y-y_{1}} \leq \frac{\phi(y+M)-\phi\left(y_{1}+M\right)}{y-y_{1}}
$$

and

$$
\frac{\phi\left(y_{2}\right)-\phi(y)}{y_{2}-y} \leq \frac{\phi\left(y_{2}+M\right)-\phi(y+M)}{y_{2}-y} .
$$

Thus,

$$
\phi\left(y_{1}+M\right)-\phi\left(y_{1}\right) \leq \phi(y+M)-\phi(y) \leq \phi\left(y_{2}+M\right)-\phi\left(y_{2}\right) .
$$

Since $\phi\left(y_{1}+M\right)-\phi\left(y_{1}\right)=\phi\left(y_{2}+M\right)-\phi\left(y_{2}\right)=0$, the proof of part 1 is complete.

2. Consider $\forall x>y_{0}$. Due to the convexity of $\phi(x)$, we have

$$
\frac{\phi(x)-\phi\left(y_{0}\right)}{x-y_{0}} \leq \frac{\phi(x+M)-\phi\left(y_{0}+M\right)}{x-y_{0}} .
$$

Thus, $\phi\left(y_{0}\right)=\phi\left(y_{0}+M\right)$ implies $\phi(x) \leq \phi(x+M)$. The convexity of $\phi(x)$ further implies that

$$
\frac{\phi(x+M)-\phi(x)}{M} \leq \frac{\phi\left(x+M^{\prime}\right)-\phi(x)}{M^{\prime}}, \forall M^{\prime} \geq M ;
$$

hence, $\phi(x) \leq \phi\left(x+M^{\prime}\right)$.

Consider $\forall x<y_{0}$. Due to the convexity of $\phi(x)$, we have

$$
\frac{\phi\left(y_{0}\right)-\phi(x)}{y_{0}-x} \leq \frac{\phi\left(y_{0}+M\right)-\phi(x+M)}{y_{0}-x} .
$$

Thus, $\phi\left(y_{0}\right)=\phi\left(y_{0}+M\right)$ implies $\phi(x) \geq \phi(x+M)$.

3. First, there must exist a point $y, y^{*}-M \leq y \leq y^{*}$ so that $\phi(y)=\phi(y+M)$. This is true because $\phi(y)$ is continuous and $\phi(y)(\phi(y+M))$ reaches the global minimum at $y^{*}\left(y^{*}-M\right.$, respectively). Hence, the uniqueness of the junction $y_{0}$ implies that $y_{0} \leq y^{*}$. Second, because $\phi\left(y^{*}-M\right)>\phi\left(y^{*}\right)$ (due to the fact that $y^{*}$ is the smallest global minimum of $\left.\phi(y)\right)$, the uniqueness of the junction $y_{0}$ implies that $y_{0}>y^{*}-M$.

Clearly, $H_{1}(y)$ is convex, and since $\pi>c, H_{1}(y)$ tends to infinity as $|y| \rightarrow \infty$. Thus, Lemma 3.2 holds for $H_{1}(y)$. Define the smallest global minimum of $H_{1}(y)$ to be $y_{1}^{*}$ and the junction between $H_{1}(y)$ and $H_{1}(y+M)$ to be $y_{1}$. The following theorem characterizes the optimal policy for the single-period inventory systems with MOQ. 
THEOREM 3.3: In the single-period problem, the optimal policy, given the initial inventory position $x$, is to order

$$
\begin{cases}y_{1}^{*}-x, & x \leq y_{1}^{*}-M \\ M, & y_{1}^{*}-M<x \leq y_{1} \\ 0, & \text { otherwise. }\end{cases}
$$

Proof: For $x \leq y_{1}^{*}-M$, the global minimum $y_{1}^{*}$ is a feasible order-up-to level; thus, it is optimal to order $y_{1}^{*}-x$. For $x>y_{1}$, since $H_{1}\left(x+M^{\prime}\right) \geq H_{1}(x), \forall M^{\prime} \geq M$ (Lemma 3.2), the optimal policy is to order nothing. For $x$ that satisfies $y_{1}^{*}-M<$ $x \leq y_{1}$, first notice that $H_{1}(x+M) \leq H_{1}(x)$ (Lemma 3.2), which implies that we should order at least $M$; second, notice that $H_{1}\left(x+M^{\prime}\right) \geq H_{1}(x+M), \forall M^{\prime}>M$, because $x+M>y_{1}^{*}$ and $H_{1}(y)$ is convex. Thus, only ordering $M$ is the optimal policy.

The optimal policy is illustrated in Figure 1. We point out that the single-period optimal policy for the model with MOQ has a different structure than the $(s, S)$ policy that is optimal for the model of fixed ordering cost and than the reorder point/batch-transfer policy that is optimal for the model of fixed batch sizes.

Now consider any time period $n, 1<n \leq N$. We first show that the following properties hold for $H_{n}(y)$.

Proposition 3.4: $H_{n}(y), n>1$ has the following properties:

1. $H_{n}(y)$ is continuous.

2. $H_{n}(y)>0$ and $H_{n}(y) \rightarrow+\infty$ as $|y| \rightarrow+\infty$.

\section{ProOF:}

1. The proof is by induction. Assume that $H_{n-1}(y)$ is continuous; then it is easily seen that $\inf \left\{H_{n-1}(y): y \geq x+M\right\}$ is continuous because

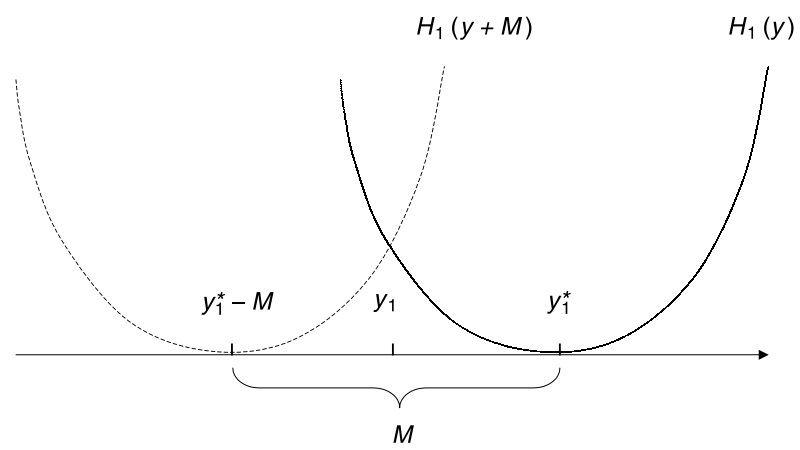

FIgURE 1. The cost function of a single-period inventory system with MOQ. 
the boundary $x+M$ is a continuous function of $x$. Thus, $V_{n-1}(x)=$ $\inf \left\{H_{n-1}(x) ; \inf \left\{H_{n-1}(y): y \geq x+M\right\}\right\}$ is continuous because the infinmum of continuous functions is continuous. Hence, $H_{n}(y)=g_{n}(y)+$ $\beta E\left(V_{n-1}\left(y-D_{n}\right)\right)$ is continuous. Finally, the continuity of $H_{1}(y)$ implies the result.

2. Notice that for $n>1$,

$$
\begin{aligned}
H_{n}(y) & =g_{n}(y)+\beta E\left(V_{n-1}\left(y-D_{n}\right)\right) \\
& =c(1-\beta) y+E\left(L\left(y, D_{n}\right)\right)+\beta E\left(U_{n-1}\left(y-D_{n}\right)+c\left(y-D_{n}\right)\right) \\
& =c y+E\left(L\left(y, D_{n}\right)\right)+\beta E\left(U_{n-1}\left(y-D_{n}\right)\right)-\beta c E\left(D_{n}\right) \\
& >E\left((h+c)\left(y-D_{n}\right)^{+}+(\pi-c)\left(D_{n}-y\right)^{+}\right)+\beta E\left(U_{n-1}\left(y-D_{n}\right)\right) .
\end{aligned}
$$

Since $E\left((h+c)\left(y-D_{n}\right)^{+}+(\pi-c)\left(D_{n}-y\right)^{+}\right)$is nonnegative and tends to positive infinity as $|y| \rightarrow \infty$ (due to $\pi>c$ ), the fact that $U_{n-1}(\cdot) \geq 0$ implies the result.

Definition 3.5: $\phi(x)$ is an M-increasing function if $\phi(x) \leq \phi\left(x+M^{\prime}\right), \forall x$ and $\forall M^{\prime} \geq M$.

Note that the regular nondecreasing functions are special cases of the $M$ increasing function for which $M=0$. The definition of the $M$-increasing functions directly implies the following proposition.

Proposition 3.6: If $\phi(x)$ and $\psi(x)$ are M-increasing functions, then the following hold:

1. $a \phi(x)+b \psi(x)$ is $M$-increasing for nonnegative $a$ and $b$.

2. $E(\phi(x-D))$ is $M$-increasing, where $E(\cdot)$ is the expectation with respect to $D$.

3. $\phi(x)$ is $M^{\prime}$-increasing with $\forall M^{\prime}>M$.

LEMMA 3.7: $V_{n}(x)$ is an M-increasing function for all $n \geq 1$.

Proof: The result holds because $V_{n}(x)=\inf _{y \in A_{x}}\left\{H_{n}(y)\right\}+\beta c E\left(D_{n}\right)$ and $A_{x+M^{\prime}} \subset$ $A_{x}, \forall M^{\prime} \geq M$.

To characterize the optimal policy for time period $n>1$, define the smallest global minimum of $H_{n}(y)$ to be $y_{n}^{*}$ (it is finite due to Proposition 3.4). Since the single-period cost functions $g_{n}(y)$ are convex and $g_{n}(y) \rightarrow+\infty$ as $|y| \rightarrow+\infty$ because $\pi>c$, we can define $y_{n, G}$ to be the unique junction between $g_{n}(y)$ and $g_{n}(y+M)$ (Lemma 3.2). Observe that if the demand is identically distributed, $y_{n, G}$ is a constant for all $n>1$; therefore, we let $y_{G}=y_{2, G}$ for simplicity.

Following the same logic as in the proof of Lemma 3.2, we can show that $H_{n}(y)$ and $H_{n}(y+M)$ must have at least one junction in $\left(y_{n}^{*}-M, y_{n}^{*}\right]$ because $H_{n}(y)$ is continuous, and $H_{n}(y)\left(H_{n}(y+M)\right)$ reaches the global minimum at $y_{n}^{*}\left(y_{n}^{*}-M\right.$, 
respectively). Let $y_{n}$ be the smallest junction between $H_{n}(y)$ and $H_{n}(y+M)$ in $\left(y_{n}^{*}-M, y_{n}^{*}\right]$ and let $\bar{y}_{n}\left(M^{\prime}\right)$ be the largest junction between $H_{n}(y)$ and $H_{n}\left(y+M^{\prime}\right)$ for $M^{\prime} \geq M$. We define $\tilde{y}_{n}=\max _{M^{\prime} \geq M}\left\{\bar{y}_{n}\left(M^{\prime}\right)\right\}$. For the ease of exposition, we simplify the notation $\bar{y}_{n}(M)$ to $\bar{y}_{n}$.

Given these definitions, we can make the following observations.

Observation 3.8:

(a) $g_{n}(y)$ is $M$-increasing for $y \geq y_{n, G}$ (i.e., $g_{n}(y) \leq g_{n}\left(y+M^{\prime}\right), \forall M^{\prime} \geq M$, $\forall y \geq y_{n, G}$ ) (Lemma 3.2).

(b) For all $y \in\left[y_{n}^{*}-M, \underline{y}_{n}\right), H_{n}(y+M)<H_{n}(y)$.

(c) Due to the asymptotic behavior of $H_{n}(y)$ (Proposition 3.4), for all $y>\tilde{y}_{n}$ we have $H_{n}(y) \leq H_{n}\left(y+M^{\prime}\right), \forall M^{\prime} \geq M$ (M-increasing).

The following relationships hold.

LEMMA 3.9: $y_{n}^{*}-M \leq \underline{y}_{n} \leq \tilde{y}_{n} \leq y_{n, G}$.

Proof: The first and second inequalities follow by the definitions of $\underline{y}_{n}$ and $\tilde{y}_{n}$. To show the third inequality, we use contradiction. Suppose $y_{n, G}<\tilde{y}_{n}$; first observe that for all $y \geq y_{n, G}, H_{n}(y)=g_{n}(y)+\beta E\left(V_{n-1}\left(y-D_{n}\right)\right)$ is $M$-increasing. This is true because $g_{n}(y)$ is $M$-increasing, $\forall y>y_{n, G}$ (Observation 3.8(a)), and $V_{n-1}(y)$ is $M$-increasing for all $y$, Proposition 3.6 implies the result. Thus, for any $y \in\left(y_{n, G}, \tilde{y}_{n}\right)$, we must have $H_{n}(y) \leq H_{n}\left(y+M^{\prime}\right), \forall M^{\prime} \geq M$. However, by the definition of $\tilde{y}_{n}$, there must exist a $M^{\prime} \geq M$ and a $y \in\left(y_{n, G}, \tilde{y}_{n}\right)$ such that $H_{n}\left(y+M^{\prime}\right)<H_{n}(y)$. This completes the proof.

Now we are ready to show the optimal policy in certain regions of the state space.

THEOREM 3.10: In the multiple-period problem, the optimal policy at time period $n>1$, given the initial inventory position $x$, is to order

$$
\begin{cases}y_{n}^{*}-x, & x \leq y_{n}^{*}-M \\ z_{n}^{*}(x)-x, & y_{n}^{*}-M<x \leq \underline{y}_{n} \\ 0, & x>\tilde{y}_{n},\end{cases}
$$

where $z_{n}^{*}(x)$ is a nondecreasing function of $x$ and $z_{n}^{*}(x)-x \geq M$.

Proof: For $x \leq y_{n}^{*}-M$, the optimal policy is obviously to order up to $y_{n}^{*}$ since the global minimum $y_{n}^{*}$ is feasible for $x$.

For $y_{n}^{*}-M<x \leq \underline{y}_{n}$, it is optimal to order up to level $z_{n}^{*}(x) \geq x+M$ because $H_{n}(y+M)<H_{n}(y)$ for all $y \in\left(y_{n}^{*}-M, y_{n}\right)$ (Observation 3.8(b)). To show that $z_{n}^{*}(x)$ is a nondecreasing function, consider $\bar{x}, x^{\prime} \in\left(y_{n}^{*}-M, \underline{y}_{n}\right]$ and assume $x<x^{\prime}$; then $\arg \min _{y \in[x+M,+\infty)} H_{n}(y) \leq \arg \min _{y \in\left[x^{\prime}+M,+\infty\right)} H_{n}(y)$.

For $x>\tilde{y}_{n}, H_{n}(y)=g_{n}(y)+\beta E\left(V_{n-1}\left(y-D_{n}\right)\right)$ is $M$-increasing (Observation $3.8(\mathrm{c}))$; thus, the optimal policy is to order nothing. 


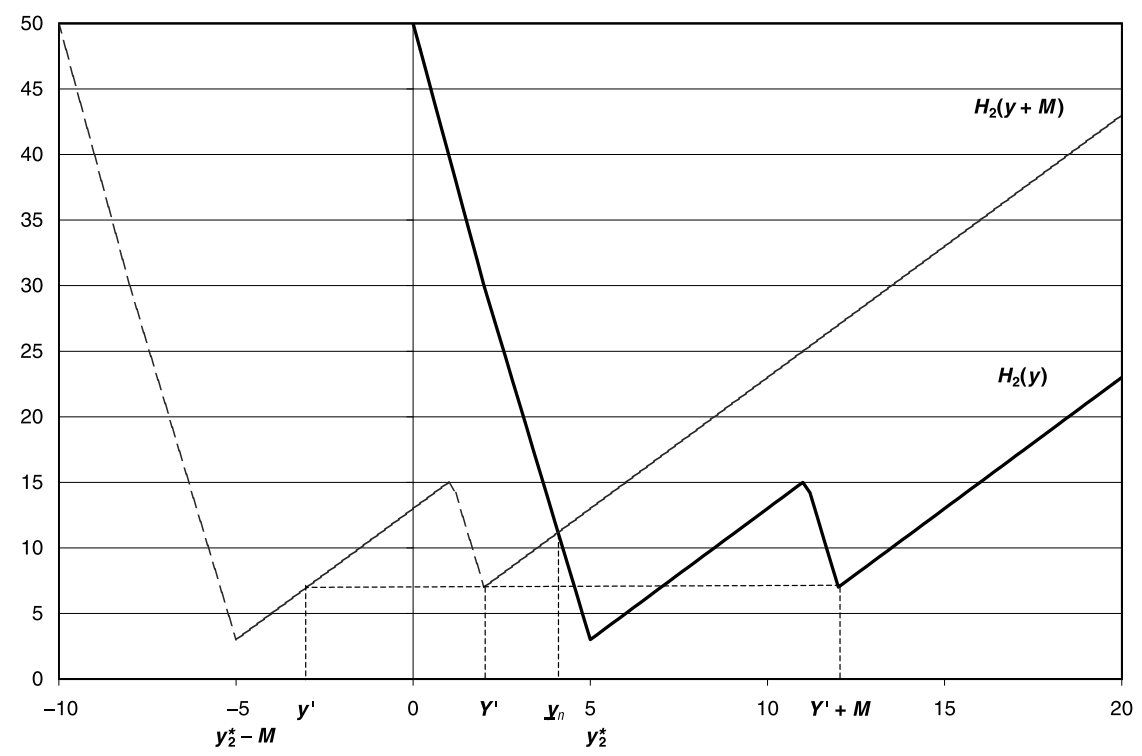

Figure 2. Example 1.

The example in Figure 2 shows that $z_{n}^{*}(x)$ might not always be equal to $x+M$ for $x \in\left(y_{n}^{*}-M, y_{n}\right]$. The example has two time periods with a deterministic demand seven units realized in the first period and a deterministic demand five units realized in the second period (the same observation can be made when the demand in the second period is also seven), and $M=10, \pi=10, h=1$, and $c=0$. See Figure 2 for the definition of $y^{\prime}$ and $Y^{\prime}$. It is obvious from Figure 2 that if $y_{2}^{*}-M<$ $x<y^{\prime}, z_{2}^{*}(x)=x+M$; however, if $y^{\prime}<x<Y^{\prime}, z_{2}^{*}(x)=Y^{\prime}+M$; and, finally, if $Y^{\prime}<$ $x<\underline{y}_{n}, z_{2}^{*}(x)=x+M$. We have examples (not reported here) with stochastic demand in which there exist multiple $y$ 's and $Y$ 's. These examples show that the optimal policies of the multiperiod systems generally have different structures than those of the single-period systems (Theorem 3.3). Together with the single-period optimal policy, these examples demonstrate that neither the optimal policy in terms of the ordering quantity nor the optimal policy in terms of the order-up-to level is monotone.

Theorem 3.10 characterizes the optimal policy in the state space except for the interval $\Omega_{n}=\left(\underline{y}_{n}, \tilde{y}_{n}\right]$. We have numerical examples (not reported here) to show that $H_{n}(y)$ has multiple junctions with $H_{n}(y+M)$; thus, $\Omega_{n}$ can be nonempty. To characterize the optimal policy in this interval, we need to answer the following question: Does there exist a unique point $y_{n}^{0} \in \Omega_{n}$ so that we should order at least $M$ for $\forall x<y_{n}^{0}$ and order nothing for $\forall x>y_{n}^{0}$ ? If there exists such a point, then $y_{n}^{0}=\tilde{y}_{n}$ because we should not order for $x>\tilde{y}_{n}$ (Theorem 3.10), and there must exist a $\epsilon>0$ so that for $x \in\left(\tilde{y}_{n}-\epsilon, \tilde{y}_{n}\right)$, we should order (due to the definition of junction and the continuity of $\left.H_{n}(y)\right)$. However, when $H_{n}(y)$ and 


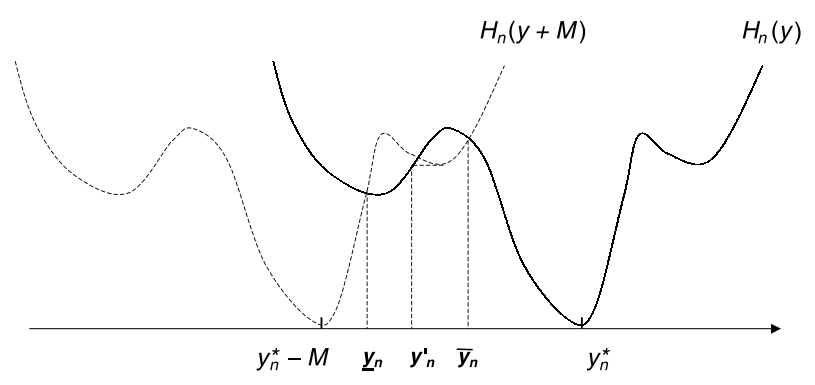

Figure 3. Example 2.

$H_{n}(y+M)$ have multiple junctions, such a point might not exist. For instance, Figure 3 illustrates a case in which $H_{n}(y)$ has three junctions with $H_{n}(y+M)$, with $\underline{y}_{n}\left(\bar{y}_{n}\right)$ being the smallest (largest, respectively) junction. If $x<\underline{y}_{n}$, we should order; if $x \in\left(\underline{y}_{n}, y_{n}^{\prime}\right)$ (see Fig. 3 for the definition of $y_{n}^{\prime}$ ), we should not order; if $x \in\left(y_{n}^{\prime}, \bar{y}_{n}\right)$, we should again order; and if $x>\bar{y}_{n}$ we should again not order. It is still not clear under what conditions such a case might exist. Thus, the existence of such a point $y_{n}^{0}$ is an open question.

We next provide bounds for $\left\{y_{n}^{*}\right\}$ as $n \rightarrow \infty$ in the case of stationary demands. Observe that Lemma 3.9 specifies upper bounds for $y_{n}^{*}, \forall n$; therefore, we focus on lower bounds. We define $y_{L}$ to be the junction between $E(L(y, D))$ and $E(L(y+$ $M, D))$. A simple heuristic policy works as follows: Order up to $y \in\left[y_{L}, y_{L}+M\right]$ if $x<y_{L}$; otherwise, do not order. It is easily seen that if the initial inventory position of a time period $x \in\left[y_{L}, y_{L}+M\right]$, the expected cost in this period is no more than $c(M+E(D))+E\left(L\left(y_{L}, D\right)\right)$. Thus, the infinite horizon long-run average cost and the total discounted cost of this heuristic policy are bounded from above for an initial state $x \in\left[y_{L}, y_{L}+M\right]$.

Proposition 3.11: For stationary demand, the smallest global minimum of $H_{n}(\cdot)$, $y_{n}^{*}$, is uniformly bounded from below for all $n$.

Proof: The proof is by contradiction. If there exists a subsequence $n_{k}$ so that $y_{n_{k}}^{*} \rightarrow-\infty$ as $n_{k} \rightarrow+\infty, H_{n_{k}}\left(y_{n_{k}}^{*}\right) \rightarrow+\infty$ (by Eq. (4) and part 2 of Proposition 3.4). Since $V_{n_{k}+1}(x) \geq H_{n_{k}}\left(y_{n_{k}}^{*}\right)+\beta c E\left(D_{n_{k}+1}\right), \forall x, V_{n_{k}+1}(x) \rightarrow+\infty, \forall x \in\left[y_{L}, y_{L}+M\right]$ as $n_{k} \rightarrow+\infty$. This creates a contradiction because the discounted cost $U_{n_{k}+1}(x)=$ $V_{n_{k}+1}(x)-c x$ is bounded by the total discounted cost of the heuristic policy.

It follows from Lemma 3.9 and Proposition 3.11 that there exists a compact set $\Omega$ so that $y_{n}^{*} \in \Omega, \forall n$. To obtain a lower bound for the limit points of $\left\{y_{n}^{*}\right\}$, we assume without loss of generality that $c=0[21]$; then $y_{L}=y_{G}, V_{n}(x)=U_{n}(x)$, and

$$
U_{n}(x)=\inf _{y \in A_{x}}\left\{E(L(y, D))+\beta E\left(U_{n-1}(y-D)\right)\right\} .
$$


Since the single-period cost function is nonnegative, Bertsekas [2] showed that $U_{n}(x) \leq U_{n+1}(x), \forall x, n$, and there exists a function $U(x)$ such that

$$
U(x)=\inf _{y \in A_{x}}\{E(L(y, D))+\beta E(U(y-D))\}
$$

In addition, for all $x, \lambda \in R$, and all $n=1,2, \ldots$, the sets $B_{n}(x, \lambda)=\{y \in$ $\left.A_{x} \mid E(L(y, D))+\beta E\left(U_{n}(y-D)\right) \leq \lambda\right\}$ are compact (Proposition 3.4 and Eq. (14)). Thus, using Proposition (1.7) of Bertsekas [2, p. 148], it follows that $U_{n}(x) \rightarrow$ $U(x)$ pointwisely as $n \rightarrow \infty$. Thus, $H_{n}(y) \rightarrow E(L(y, D))+\beta E(U(y-D))$ pointwisely (by the monotone convergence theorem). Define $H(y)=E(L(y, D))+$ $\beta E(U(y-D))$. $H(y)$ satisfies Proposition 3.4 because $H_{n}(y) \rightarrow H(y)$ pointwisely as $n \rightarrow \infty$ and $U_{n}(\cdot) \geq 0, \forall n$. Finally, in $\Omega, H_{n}(y)$ converges uniformly to $H(y)$ because $\Omega$ is compact.

Lemma 3.12: Let $y^{*}$ be the smallest global minimum of $H(y)$; then $y^{*} \geq y_{G}$.

Proof: For all $x \in S$,

$$
\begin{aligned}
U(x) & \geq H\left(y^{*}\right) \\
& =E\left(L\left(y^{*}, D\right)\right)+\beta E\left(U\left(y^{*}-D\right)\right) \\
& \geq E\left(L\left(y^{*}, D\right)\right)+\beta E\left(L\left(y^{*}, D\right)\right)+\cdots \\
& =\frac{E\left(L\left(y^{*}, D\right)\right)}{1-\beta} .
\end{aligned}
$$

Since the heuristic policy has a long-run average cost less than or equal to $E\left(L\left(y_{G}, D\right)\right)$, for a $x \in\left[y_{G}, y_{G}+M\right]$,

$$
\frac{E\left(L\left(y^{*}, D\right)\right)}{1-\beta} \leq U(x) \leq \frac{E\left(L\left(y_{G}, D\right)\right)}{1-\beta} .
$$

To show that $y^{*} \geq y_{G}$, we observe that if $y^{*}<y_{G}$, then $E\left(L\left(y^{*}, D\right)\right)>E\left(L\left(y_{G}, D\right)\right)$ from the definition of $y^{*}$, the convexity and asymptotic behavior of $E(L(y, D))$. Thus, (7) is violated.

Now we are ready to provide a lower bound for the limit points of $\left\{y_{n}^{*}\right\}$.

THEOREM 3.13: $\underline{\lim }_{n \rightarrow \infty} y_{n}^{*} \geq y^{*}$.

Proof: The proof is by contradiction. Consider a limit point $\hat{y}$ of $\left\{y_{n}^{*}\right\}$; that is, there exists a subsequence $n_{k}$ so that as $n_{k} \rightarrow \infty, y_{n_{k}}^{*} \rightarrow \hat{y}$. Suppose $\hat{y}<y^{*}$. Because $y^{*}$ is the smallest global minimum of $H(\cdot)$, we must have

$$
H(\hat{y})>H\left(y^{*}\right) .
$$


On the other hand, the definition of $y_{n_{k}}^{*}, \forall n_{k}$, implies that

$$
H_{n_{k}}\left(y_{n_{k}}^{*}\right) \leq H_{n_{k}}\left(y^{*}\right) \text {. }
$$

Let $n_{k} \rightarrow \infty$; first we have

$$
H_{n_{k}}\left(y^{*}\right) \rightarrow H\left(y^{*}\right)
$$

due to the pointwise convergence of $H_{n}(\cdot)$. Second, we observe that

$$
\left|H(\hat{y})-H_{n_{k}}\left(y_{n_{k}}^{*}\right)\right| \leq\left|H(\hat{y})-H\left(y_{n_{k}}^{*}\right)\right|+\left|H\left(y_{n_{k}}^{*}\right)-H_{n_{k}}\left(y_{n_{k}}^{*}\right)\right| .
$$

As $n_{k} \rightarrow \infty$, the first term on the right-hand side converges to zero because $H(\cdot)$ is continuous; the second term on the right-hand side also converges to zero because $H_{n_{k}}(\cdot)$ uniformly converges to $H(\cdot)$ in $\Omega$. Thus, we must have

$$
H_{n_{k}}\left(y_{n_{k}}^{*}\right) \rightarrow H(\hat{y})
$$

Inequality (9) and relations (10) and (11) imply $H(\hat{y}) \leq H\left(y^{*}\right)$. This completes the proof by contradiction.

In the case of stationary demand, it follows from Theorem 3.13 and Lemmas 3.9 and 3.12 that the interval $\left[y_{n}, \tilde{y}_{n}\right]$ is asymptotically bounded by $\left[y_{G}-M, y_{G}\right]$, where $y_{G}$ can be easily determined from the known single-period cost function. Furthermore, $\tilde{y}_{n}$ is asymptotically bounded from above by $y_{n}^{*}$. Therefore, Theorem 3.10 implies that asymptotically it is optimal to stop ordering above a certain point smaller than or equal to $y_{n}^{*}$.

\section{CONCLUSION}

In this article, we partially characterized the optimal inventory policy for the singleproduct, periodic-review inventory systems in which the ordering cost is linear but the order size must be either zero or larger than or equal to a minimum order quantity. There are many interesting research topics left unexplored for the stochastic inventory systems with minimum order quantity. For instance, we still do not know whether there exists a unique boundary between the ordering region and the nonordering region; nor do we know the existence of a stationary optimal control policy in the infinite time horizon under the average cost criterion; finally, given the complex structure of the optimal policy, easily implementable and effective heuristic policies need to be developed for the inventory systems with either a single product or multiple products sharing the minimum order quantity.

\section{Acknowledgment}

This research was supported in part by a Faculty Research Grant from Rutgers Business SchoolNewark and New Brunswick. 


\section{References}

1. Axsater, S. (1993). Continuous review policies for multi-level inventory systems with stochastic demand. In S. Graves, A. Rinnooy Kan, \& P. Zipkin (eds.), Logistics of production and inventory. Amsterdam: Elsevier (North-Holland).

2. Bertsekas, D.P. (1995). Dynamic programming and optimal control, Vol. 2. Belmont, MA: Athena Scientific.

3. Chan, E.W. \& Muckstadt, J.A. (1999). The effects of load smoothing on inventory levels in a capacitated production inventory system. Technical Report, School of Operations Research and Industrial Engineering. Cornell University, Ithaca, NY.

4. Chen, F. (2000). Optimal policies for multi-echelon inventory problems with batch ordering. Operations Research 48: 376-389.

5. De Bodt, M.A. \& Graves, S.C. (1985). Continuous-review policies for a multi-echelon inventory problem with stochastic demand. Management Science 31: 1286-1299.

6. Federgruen, A. \& Katalan, Z. (1996). The stochastic economic lot scheduling problem: Cyclical base-stock policies with idle times. Management Science 42: 783-796.

7. Fisher, M. \& Raman, A. (1996). Reducing the cost of demand uncertainty through accurate response to early sales. Operations Research 44: 87-99.

8. Gallego, G. \& Wolf, A.S. (2000). Capacitated inventory problems with fixed order costs: Some optimal policy structure. European Journal of Operational Research 126: 603-613.

9. Heyman, D. \& Sobel, M. (1984). Stochastic models in operations research. Vol. 2. New York: McGraw-Hill.

10. Karlin, S. (1958). Optimal inventory policy for the Arrow-Harris-Marschak dynamic model. In K.J. Arrow, S. Karlin, and H. Scarf (eds.), Studies in the Mathematical Theory of Inventory and Production. Stanford, CA: Stanford University Press.

11. Karmarkar, U. (1987). Lot sizes, lead-times and in-process inventories. Management Science 33: 409-419.

12. Katehakis, M. \& Bradford, P. (2002). Contract constrained resource allocation. In INFORMS Annual Meeting, San Jose, CA.

13. Lee, H.L. \& Moinzadeh, K. (1987). Two-parameter approximations for multi-echelon repairable inventory models with batch ordering policy. IIE Transaction 19: 140-149.

14. Porteus, E.L. (1971). On the optimality of generalized (s,S) policies. Management Science 17: 411-427.

15. Ross, S.M. (1970). Applied probability models with optimization applications. New York: Dover.

16. Scarf, H. (1960). The optimality of $(\mathrm{s}, \mathrm{S})$ policies in dynamic inventory problems. In K. Arrow, S. Karlin, \& P. Suppes (eds.), Mathematical models in the social sciences. Stanford, CA: Stanford University Press.

17. Sethi, S.P. \& Cheng, F. (1997). Optimality of (s, S) policies in inventory models with Markovian demand. Operations Research 45: 931-939.

18. Song, J.S. \& Yao, D.D. (2001). Supply chain structures: Coordination, information and optimization. Boston: Kluwer Academic.

19. Veinott, A.F. (1965). The optimal inventory policy for batch ordering. Operations Research 13: $424-432$.

20. Veinott, A.F. (1966). On the optimality of (s,S) inventory policies: New conditions and a new proof. SIAM Journal of Applied Mathematics 14: 525-552.

21. Veinott, A.F. \& Wagner, H. (1965). Computing optimal (s,S) inventory policies. Management Science 11: 525-552.

22. Zheng, Y.S. \& Federgruen, A. (1991). Finding optimal (s, S) policies is about as simple as evaluating a single policy. Operations Research 39: 654-665.

23. Zipkin, P. (2000). Foundations of inventory management. Boston: McGraw-Hill. 